\title{
IDENTITÉ ET RÉSISTANCE: FONDEMENTS \\ ET ENJEUX PHILOSOPHIQUES
}

Jean-Pierre Zarader

Résister. Le terme possède son aura romantique et ses lettres de noblesse. Mais résister à quoi, ou à qui ? La résistance est toujours résistance à une hégémonie ou à une homogénéisation. Et aujourd'hui plus que jamais, à l'ère de la globalisation et de l'industrie culturelle, aux deux à la fois, indissolublement liées, ce que Derrida nomme avec justesse une homohégémonie. Ce mot valise exprime en un sens tout mon propos: qu'une puissance hégémonique, dominante, a toujours tendance à homogénéiser, c'est-à-dire à rendre homogène, à instaurer voire à imposer une uniformité. On pressent déjà que le destin d'une telle volonté homohégémonique sera de se heurter à ce qui demeure, voire entend bien demeurer, hétérogène, en d'autres termes à une altérité qui résiste à une telle volonté hégémonique et homogénéisante. Cette dernière peut être celle de l'Etat, du politique, comme elle peut être celle de l'économie. Mais qu'il s'agisse de l'ordre politique ou de l'ordre économique, de l'ordre étatique ou de l'ordre mondial, ce contre quoi se dresse la résistance, c'est la figure de la maîtrise. Une figure qui est aussi vieille que la philosophie elle-même - comme la figure de la résistance.

C'est dans le domaine de la culture que l'identité peut apparaître comme le résultat d'un effort, jamais achevé, de résistance. Pourtant, par delà les cas particuliers, à la fois historiques et exemplaires que chacun pourrait citer, la notion de résistance, associée à celle d'identité, possède une dimension philosophique qui est comme la matrice logique ou conceptuelle des figures historiques qui pourront être évoquées. On pourrait dire que tout commence avec Hegel, puisque toute la philosophie contemporaine s'est construite en entrant en résistance contre le discours dominant et totalisant que fût (ou que l'on a cru être) celui de la philosophie de Hegel. C'est que la philosophie hégélienne n'est pas une philosophie parmi d'autres. Elle s'est voulue achèvement de toute l'histoire de la pensée, et même, en un sens, de l'histoire tout court, avènement d'une rationalité universelle à laquelle rien ne pouvait échapper: une philosophie de la maîtrise absolue.

Tout commence donc avec la capacité reconnue au concept hégélien, à ce que Hegel nomme le concept, de comprendre en lui - 
dans les deux sens du terme: à la fois englober et rendre compte de comprendre en lui toute réalité. Cette prétention hégémonique du concept s'exprimerait dans l'affirmation célèbre: "Tout ce qui est rationnel est réel (effectif); tout ce qui est réel (effectif) est rationnel". On comprend que l'hégélianisme ait pu apparaitre comme un panlogisme, c'est-à-dire comme l'affirmation résolue de la victoire de la raison sur toute irrationalité, la victoire de l'universel sur toute particularité. Encore conviendrait-il, pour rendre justice à Hegel, de rappeler que la raison hégélienne n'est pas une rationalité étroite, un pur rationalisme positiviste: l'universel hégélien n'est pas un universel abstrait qui refuserait toute particularité, toute différence, toute altérité. Bien au contraire, puisque ce que Hegel nomme le concept est à entendre comme un processus qui, par son propre dynamisme, sa propre vie pourrait-on dire, se saisit de tout ce qui n'est pas lui, de tout ce qui lui est étranger et différent, pour se l'approprier - l'absorber et le résorber -, s'en nourrir, en faire le corps de sa propre identité à laquelle dès lors rien ne pourrait être étranger - mais rien aussi, bien sûr, ne pourrait résister. Telle est cette raison dont Hegel a pu montrer l'omniprésence ou la parfaite maitrise dans tous les domaines de la réalité, y compris dans l'histoire. Et ce n'est pas un hasard si on a donné à un recueil de cours de Hegel le titre: $L a$ raison dans l'histoire: l'esprit du monde est l'universel qui se réalise dans et par l'histoire des différents peuples particuliers.

C'est contre une telle prétention, une telle hégémonie de la raison (donc de l'universel - et l'on pourrait ajouter: de l'Occident), que la philosophie contemporaine est entrée en résistance. Une résistance qui a pu revêtir des modalités diverses selon les auteurs, mais qui s'est toujours faite dans le but de résister à cette totalité hégélienne soupçonnée d'être totalitaire et accusée de méconnaître le moment de la différence, ou plus exactement la différence (ou l'altérité) pour ellemême et non comme simple moment - précisément - d'un procès conceptuel, qui l'accueillerait, certes, mais pour mieux la dépasser, en assurer la relève (Aufhebung). Ou, pour le dire autrement: la philosophie contemporaine n'a cessé de tenter de penser ce qui échappe à la pensée et à son effort de totalisation, et l'on comprend pourquoi la notion d'événement joue un rôle fondamental dans ces philosophies. L'événement, c'est ce qui défie toute prévision, ce qui demeure irréductible à l'effort de totalisation, ce qui excède le concept. L'événement, dans son surgissement même, est donc ce dont la pensée ne saurait rendre compte, ce qui essentiellement résiste - ce que je serais tenté de nommer le cri du monde. 
Mais remonter ainsi vers la source logique des notions d'identité et de résistance, c'est reconnaître que la notion d'identité est suspecte pour la philosophie. Suspecte dans la mesure même où, et cela bien avant Hegel, depuis l'origine, depuis Platon, l'identité est conçue comme étant du côté d'un refus de la différence, du refus de l'Autre. C'est dire que l'identité est conçue (ou a été le plus souvent conçue) comme identique à elle-même, compacte, "en soi", alors qu'elle est toujours double et scindée, qu'elle est toujours, pour être une identité vivante, vive, autre qu'elle-même, différente d'ellemême, dans tous les sens du terme. Un terme dont Jacques Derri$\mathrm{da}$ - et il écrit alors différance, avec un $a$ - a souligné la polysémie: l'acte de différer de soi, dans le temps, de ne jamais coïncider avec soi, mais également l'opposition à soi, le différend. Bref l'identité s'est le plus souvent voulue une, alors qu'elle ne peut être que plurielle. Et cela est vrai, bien sûr, de toute identité - que ce soit celle d'une culture ou celle d'un individu: toute culture nait d'un héritage qu'elle ne peut s'approprier qu'en le métamorphosant profondément, et elle ne pourra elle-même survivre qu'en acceptant d'être à son tour métamorphosée, altérée, si l'on veut, mais maintenant dans cette altération même son identité vive. Je pense bien sûr à Malraux qui écrivait: "Il n'y a que les œuvres mortes qui ne changent pas". " C'est en ce sens précis que Derrida (à la suite de Walter Benjamin) parlera du survivre: non seulement continuer à vivre, mais vivre au-dessus et davantage. J'y reviendrai dans un instant en évoquant l'importance de la traduction et donc de la pluralité des langues. On comprend pourquoi l'identité est indissociable de ce que Derrida nomme une exappropriation: affirmer son identité c'est, en un sens, affirmer sa maîtrise, mais c'est aussi, contradictoirement, désirer être délogé de cette maîtrise, être visité par l'autre - dans cette hospitalité de visitation que Derrida distingue soigneusement de la simple hospitalité d'invitation. Toute identité doit pouvoir être volée, en partie, pour survivre - pour survivre autrement, en ou par autre chose. Altérité/altération: la nuance qui sépare ces deux termes exprime bien la distinction qui existe entre ces deux types d'hospitalité.

C'est donc volontairement que j'emploie ici le terme d'altération et non celui, plus doux et moins effrayant, d'altérité. Pour clarifier la distinction que je propose ici, je dirais que l'altérité, c'est l'autre, alors que l'altération, c'est l'autre en tant qu'il m'entame, qu'il me modifie, qu'il me change, peut-être qu'il mabîme. Mais que serait une identité qui se prétendrait ouverte à l'autre, à
"(MALRAuX, André. La Tête d'Obsidienne Paris: Gallimard, 1974: 253.) 
* (TOURNIER, Michel. Vendredi ou les limbes du Pacifique. Paris: Gallimard, 1967: 12.)

*(DERRIDA, Jacques. Le monolinguisme de l'autre. Paris: Galilée, 1996: 78.) l'altérité, et qui en même temps refuserait de se laisser entamer par l'autre? Ce serait une identité qui serait en réalité fermée sur ellemême, enfermée en soi, une identité morte puisqu'elle refuserait toute ouverture, refuserait par avance toute altération. Si la chose est vraie de l'identité d'une culture, chacun sait à quel point cela est vrai également d'une identité biologique. Pour le dire très simplement, il n'y a pas de pureté, il n'y a que des mélanges. Les philosophes disent: ce qui est premier, c'est la relation - et celle-ci précède les termes mêmes de la relation. Il n'y a donc pas de pureté, mais il y a, hélas, le phantasme toujours renaissant de la pureté - d'une origine sans tache. On me permettra donc de rappeler ici le conseil que le capitaine Van Deyssel donne à Robinson: "Crusoé, lui-ditil, écoutez-moi bien: gardez-vous de la pureté. C'est le vitriol de l'âme." $\mathrm{Ou}$, plus conceptuellement, la réflexion de Jacques Derrida, qui voit précisément dans le refus d'une telle pureté l'acte même de la déconstruction: “je n'ai jamais cessé de remettre en question le motif de la pureté [...] le premier mouvement de ce qu'on appelle la déconstruction la porte vers cette critique du phantasme ou de l'axiome de la pureté ou vers la décomposition analytique d'une purification qui reconduirait à la simplicité indécomposable de l'origine."* Or c'est cette altérité ou cette altération que la philosophie a souvent voulu écarter ou annexer, en un mot réduirecomme on dit fort bien: "réduire au silence."

Je rappellerai donc simplement pour mémoire que Platon, notamment dans le Phèdre, opère une critique du discours écrit contredistingué du discours oral. L'oralité à laquelle Socrate ne veut pas renoncer, voyant dans ce pharmakon ${ }^{1}$ qu'est l'écriture, moins un remède à l'oubli, qu'un poison de la mémoire, renvoie ici à cette figure de la maîtrise, cette métaphysique de la présence (présence du sens à la conscience - à laquelle rien ne saurait échapper) que Derrida n'a cessé de critiquer. Le discours écrit est comparé à un orphelin privé de l'assistance de son père: le texte, contrairement au discours oral, se trouverait ainsi livré à lui-même, délié de tout sens premier, de tout "vouloir dire" originel, exposé aux interprétations les plus hasardeuses. Cette condamnation de l'écriture est donc emblématique d'une volonté de maîtrise absolue qui ne peut asseoir son empire et/ou son emprise qu'en refusant toute altérité, tout étrangèreté. Pureté qui prétendrait refuser toute scorie. Ce

\footnotetext{
${ }^{1}$ Le grec Pharmakon désigne à la fois, en une équivocité proprement spéculative, le remède et le poison.
} 
qui fait peur ici, c'est l'altérité, la différence/différance - c'est elle que de tout temps la figure de la maîtrise a voulu conjurer, et c'est elle qui entre en résistance.

Qu'il me soit permis de rappeler que c'est sans doute cette altérité, cette contingence, cette impureté, ce cri du monde, qu'Adorno (préférant les partitions aux interprétations) rejette dans le jazz, qui ne vit que de ses différentes interprétations, de ses différents écarts à l'égard de standards - et qui ignore superbement les partitions. ${ }^{*} S$ 'il est vrai, comme l'écrit Patrick Williams, que "Le jazz introduit la contingence au cœur de l'œuvre et acquiesce à l'absence de clôture entre création artistique et vie quotidienne, [s'il est vrai qu'] il est cette musique qui s'ouvre à l'humeur des hommes et au temps qu'il fait", comment ne pas voir et entendre que le jazz nous apprend, pour peu qu'on accepte de l'entendre, le refus de toute clôture, l'Ouverture.

J'ignore si cet exemple du jazz est pertinent, mais je voudrais prendre un second exemple qui est sans doute, pour l'apprenti philosophe, moins risqué - et qui est en un sens plus fondamental: celui de la langue. N'étant pas historien, il ne m'appartient pas de dire si - pour ne citer ici qu'un cas - l'arabisation des populations berbères (notamment en Kabylie) a été un enjeu politique majeur et si les colonisateurs français n'ont pas fortement contribué à cette arabisation, dans la mesure où les kabyles, en Algérie, étaient de farouches opposants à la colonisation. Nier ou s'efforcer de nier la langue d'un peuple ou d'une population, c'est nier ou s'efforcer de nier ce peuple - et en favorisant l'arabisation des berbères, jusque dans leurs noms propres, on peut se demander si la puissance coloniale n'entendait pas réduire au silence, dans tous les sens $\mathrm{du}$ terme, les kabyles d'Algérie. Mais ce qui m'importe, d'un point de vue philosophique, c'est de souligner combien l'imposition d'une langue (unique) et donc la réduction de la pluralité des langues est toujours un facteur d'appauvrissement, non seulement pour la langue réduite au silence mais même pour celle qui, figure suprême de la maîtrise, parvient à s'imposer ou à imposer sa domination. Et cela dans la mesure même où la traduction, l'exercice et l'épreuve de la traduction, est un enrichissement des deux langues. Une telle épreuve de la traduction est, comme l'a souligné Jacques Derrida à la suite de Walter Benjamin, une condition de la survie des œuvres. C'est que toute traduction permet à un texte de survivre dans le double sens du terme: vivre plus longtemps et vivre davantage, à un niveau plus élevé. Dans la traduction, en effet, l'original n'est pas restitué à l'identique, comme on le croit naïvement, mais
* (Relire WILLIAMS, Patrick. "Le déni d'Adorno". L'Homme, 175-176. Paris: Editions EHESS, 2005: 219-226.) 
* (BENJAMIN, Walter. Oeuves I. "La tâche du traducteur", trad. M. de Gandillac, revue par Rainer Rochlitz. Paris: Gallimard, "Folio essais": 49,260 .) il est doté d'un surcroît de vie et de sens: "Car dans sa survie, écrit Benjamin, qui ne mériterait pas ce nom si elle n'était mutation et renouveau du vivant, l'original se modifie [...] La traduction d'un texte sera en vérité un moment de sa propre croissance [...] La tâche du traducteur est d'élargir et d'approfondir sa propre langue au moyen de la langue étrangère." Ainsi la traduction est-elle utile à la fois à la langue d'origine et à la langue du traducteur: elle est vie et survie des œuvres et des langues. L'imposition d'une langue - identique pour tous - est une clôture doublement mortifere. Où l'on retrouverait le vieux désir ou le vieux démon d'un retour à la pureté, à une pureté originelle, la langue-mère d'avant la chute dans la pluralité des langues: le vieux rêve d'une tour de Babel d'avant la destruction et la dispersion. Le vieux démon de l'identité.

Il n'y a donc nulle contradiction à affirmer que l'identité se constitue dans la résistance et que la résistance elle-même est toujours une résistance à l'identité. Cette identité qui se constitue dans la résistance, c'est ce que les philosophes nomment la différence et elle se fait toujours contre l'identité qui entend réduire toutes les différences, qui entend tout homohégémoniser.

On comprend en quel sens toutes les cultures singulières sont hantées par la menace d'assimilation - comme si l'ouverture aux autres (à laquelle, il faut bien le reconnaitre, l'Occident s'est un peu - trop - vite identifié), sous couleur d'exotisme ou d'universalisme, n'était que l'autre nom de l'ethnocentrisme, voire de l'ethnocide. La résistance est donc bien refus de l'assimilation, même si elle n'est pas pour autant et nécessairement sécession, refus pur et simple - ce qui la renverrait alors à une clôture sur soi et ferait d'elle le contraire même de ce qu'elle est ou veut être. Identité close, elle n'aurait plus alors que le nom, usurpé, de résistance. La véritable résistance est l'effort constant, insistant, pour empêcher une culture - la culture dominante - de se replier sur elle-même en refusant les différences dans l'acte même par lequel elle semble les reconnaître, et c'est ainsi qu'elle est elle-même affirmation d'une identité ouverte.

C'est bien en ce sens que la philosophie contemporaine a tendance à considérer que la résistance, c'est le refus de l'Un, de l'identité close sur elle-même. C'est que, comme le disait Jean-Luc Godard, en une phrase qui saisit à elle seule l'essence même du montage: "Il n'y a pas d'image, il n'y a que des images [...] quand il y en a deux, il y en a trois." Il n'y a donc jamais la culture, une et refermée sur elle-même, il n'y a que des cultures - et de leur seule coexistence naît quelque chose qui les dépasse. 
L'unité ouverte par excellence, qui est aussi l'identité par excellence, c'est la langue (ce qui ne veut pas dire, bien sûr, que la langue ne puisse être l'instrument d'une domination et d'un asservissement - bref, la figure même de la maitrise et de la clôture). Jacques Derrida écrit ainsi, évoquant l'unité d'une langue: "Cette unité ne se compare à aucune autre. Elle est accessible à la greffe la plus radicale, aux déformations, aux transformations, à l'expropriation." C'est cette unité ouverte que Derrida nomme écriture, même si, comme il le souligne lui-même, il peut s'agir de l'oral, du vocal, du musical (et on pourrait ajouter: le pictural, le sculptural). C'est dire aussi que toute tradition doit être reprise - c'est-à-dire, entrer dans un processus de répétition mais non pas à l'identique (car cela signerait la mort de cette tradition, sa mortification ou sa momification), mais avec un écart, une répétition dans la différence, une différance, ce que Derrida nomme l'itérabilité: une logique qui lie la répétition à l'altérité.

Cette brève généalogie des concepts d'identité et de résistancede la relation agonistique des deux termes - pourrait être illustrée par la tension qui existe, en art, entre le Musée Imaginaire, qui est une création occidentale, et les différentes œuvres qu'il accueille ou annexe.

Je laisserai donc de côté les grandes figures historiques de la résistance et même leur très lointain ancêtre, la figure d'Antigone, le premier grand "Non" de l'humanité - dont on ne soulignera jamais assez qu'il est celui d'une femme. Très loin semble-t-il du domaine politique, je voudrais évoquer la résistance telle qu'elle est à l'œuvre dans le domaine de l'art. Et d'abord, en deux mots, la résistance telle qu'elle se trouve thématisée dans les Ecrits sur l'art d'André Malraux, sous la figure de ceux qu'il nomme les "génies réfractaires." Génies réfractaires: tous ceux qui résistent à leur assimilation par le Musée Imaginaire (Goya, Breton, Picasso, la sculpture africaine - les masques). Car tout l'effort de Malraux, dans les Ecrits sur l'art, est à la fois de souligner le caractère réfractaire de certaines œuvres (en insistant donc sur ce qui en elles résiste, sur leur caractère proprement exorbitant) et de montrer que toute crainte d'une "récupération" de ces œuvres par le Musée Imaginaire est sans objet dans la mesure où - en entrant au Musée - ces œuvres réfractaires modifient, métamorphosent le Musée lui-même.

Ce qui se joue ici, dans le rapport du Musée Imaginaires aux œuvres réfractaires, c'est bien le rapport de l'universel (le Musée Imaginaire, l'Occident, la maîtrise) et de la particularité (les diffé-
* (DERRIDA, Jacques. Le monolinguisme de l'autre. op. cit.: 123-124.) 
* (SPENGLER, Oswald. Le déclin de l'Occident, première partie, trad. $\mathrm{M}$. Tazerout. Paris: Gallimard, 1976: 35.)

* (DERRIDA, Jacques. L'autre cap. Paris: Minuit, 1991: 68.) rentes œuvres annexées - ou plus précisément: les oeuvres annexées, dans leur différence). Autant dire, encore et toujours, le rapport du Même et de l'Autre, de l'identité et de la résistance.

C'est donc le statut même de l'universel qui est en jeu, ou pour le dire de manière plus radicale encore, c'est l'universalisme occidental qui est ici en question - et cette question occupe tout le vingtième siècle. Celui-ci s'ouvre sur la critique bien connue de Spengler pour qui la "validité universelle est une inférence toujours fausse de soi sur autrui"* mais aussi sur l'interrogation de Valéry qui feignait de s'étonner que la particularité des Français soit de se croire, de se sentir "universels - je veux dire: hommes d'univers... [d'] avoir pour spécialité le sens de l'Universel”, enfin sur Husserl qui, quelques années plus tard, affirmait avec assurance que les philosophes européens, en tant qu'ils se consacrent à la raison universelle, sont les "Fonctionnaires de l'Humanitê".

Mais le vingtième siècle ne s'ouvre pas seulement sur cette question, il se clôt sur elle-même si celle-ci s'est approfondie et comme retournée. Jacques Derrida pourrait en témoigner, qui n’a cessé de tenter un mouvement de résistance à l'universalisme européen sans abandonner tout à fait celui-ci: “C'est une logique, la logique même, que je ne veux pas ici critiquer. Je serais même prêt à y souscrire: mais d'une main seulement, car j'en garde une pour écrire ou pour chercher autre chose, peut-être hors d'Europe [...] pour ne pas fermer d'avance une frontière à l'à-venir de l'événement, à ce qui vient, à ce qui vient peut-être et peut-être vient d'une tout autre rive.”* Cette "autre rive”, c'est bien sûr l'autre rive de la Méditerranée, celle dont nous vient précisément la culture berbère.

Plus fondamentalement encore, on pourrait souligner que l'œuvre d'art elle-même a pu être pensée en termes de résistance. Résistance non seulement au pouvoir politique, à la société, à la mode etc., mais à sa propre identité close sur elle-même, à cette autonomie si chèrement conquise (contre les dieux, la beauté, et même le "sujet") et soulignée voire célébrée aussi bien par Merleau-Ponty que par Malraux et Blanchot. Résistance, donc, à une complaisance à soi, à un repli narcissique sur soi, à une ivresse de la pure immanence. L'espace qui m'est imparti me contraint à juxtaposer (art, politique, ontologie) ce qui forme en réalité une unité. Je me bornerai donc ici à une simple indication. Que toute résistance, quelque forme qu'elle prenne, soit une résistance à l'ivresse ou à la folie de la pure immanence, on s'en convaincra en relisant le beau livre de Jean-Luc Nancy, La communauté désouvrée: 
"La communauté résiste: en un sens, je l'ai dit, elle est la résistance elle-même [...] La communauté résiste à l'immanence infinie".* Cette résistance - rapportée à la création artistique - consiste alors à reconnaître une extériorité, un dehors, ce qui en l'œuvre excède l'œuvre. Cette extériorité peut être celle d'un sens transcendant: l'empreinte du sacré, ce que Benjamin appelait la valeur cultuelle, l'aura, par opposition à la valeur d'exposition. Mais cette extériorité peut également être, à même la matière ou la matérialité de l'œuvre, l'extériorité d'éléments non esthétiques ou non immédiatement esthétiques: l'intrusion dans la peinture de fragments de végétaux ou d'animaux (le papillon naturel - comme le note Breton - qui fait son apparition dès 1933 dans un tableau de Picasso). Procédé du montage ou du collage qui peut être lu - et cela montre bien la complexité du problème - aussi bien comme puissance de la peinture (ou pour John Cage, puissance de la musique) et donc d'une identité sûre d'elle-même et dominatrice, que comme humilité d'une peinture contrainte de s'ouvrir à ce qui n'est pas elle, à ce qui lui résiste (le réel non esthétique - jusqu’à l'excrément et les différentes humeurs du corps).

On ne peut me semble-t-il comprendre de telles pratiques esthétiques que si l'on comprend qu'en elles se joue l'éternel conflit du Même et de l'Autre, de l'identité et de la différence, de l'œuvre et du monde. En d'autres termes, ce qui est en jeu ici, c'est bien le problème de l'identité et celui de la résistance. L'enjeu est d'ouvrir l'œuvre à tout ce qui n'est pas elle - et qui entend même la nier: le papillon comme le végétal, évoqués précédemment, sont ici, de toute évidence, une figure de l'éphémère - et qui comme tels résistent à la pure idéalité avec laquelle l'art a pu se confondre. Il s'agit donc bien d'entrer en résistance contre cette complaisance à soi, cette superbe de l'art - de l'Art avec une majuscule - qui prétendrait (qui prétendait) ériger en absolu une Beauté que le monde dans son opacité et dans la violence qui fut la sienne, notamment au XXe siècle, ne pouvait que dénoncer et accuser de mensonge. Car c'est bien toute une conception classique et romantique de l'art que l'art contemporain, entrant en résonance avec l'art des plus hautes époques, ébranle - et qu’il ébranle précisément en laissant apparaître tout ce qui lui résiste ou la dénonce. Et sans doute pourraiton aller encore plus loin et affirmer que toute véritable création est résistance puisque, d'un point de vue purement esthétique, le style pourrait représenter cette figure de la maitrise contre laquelle 
* (ADORNO, Theodor; HORKHEIMER, Max. La dialectique de la raison. trad. Eliane Kaufholz. Paris: Gallimard, 1974: 139.)
Palavras-chave: resistência; identidade; alteridade; língua; cultura; arte. les plus grands artistes sont entrés en résistance - y compris donc, comme ce fut manifestement le cas avec Picasso, résistance contre eux-mêmes lorsqu'ils étaient en danger d'incarner un style. C'est ainsi que Adorno et Horkheimer peuvent écrire: "Lidée d'un style comme cohérence purement esthétique est un rêve romantique tourné vers le passé [...] Même les œuvres que l'on définit comme classiques, comme la musique de Mozart, contiennent des tendances objectives qui contrastent avec le style qu'elles incarnent. Jusqu’à Schönberg et Picasso, les grands artistes ont conservé leur méfiance à l'égard du style, ils s'en sont moins tenus au style qu'à la logique de l'objet". . C'est dire que la création ne peut être pensée qu'en termes de résistance - c'est là son unique, et précaire, identité.

\section{Jean-Pierre Zarader}

Professor de filosofia do Liceu Saint-Martin de France, e pesquisador associado da Sorbonne-Nouvelle (Paris III). Autor de Malraux ou la pensée de l'art (Paris: Ellipses, 1998) e do Vocabulaire de Malraux (Paris: Ellipses, 2001), publicou ainda: Philosophie et cinéma (Paris: Ellipses, 1997), "Philippe de Broca, cinéaste de l'apparence", in Philippe de Broca, (Paris: Henri Veyrier, 1990), Robinson philosophe: Vendredi ou la vie sauvage de Michel Tournier, un parcours philosophique -suivi de "Le philosophe aux images", un entretien avec Michel Tournier (Paris: Ellipses, 1999). Matrix, Machine Philosophique, (em colaboração com Alain Badiou, T. Benatouil, E. During, P. Maniglier, D., Rabouin) (Paris: Ellipses, 2003).

\section{Resumo}

A resistência é sempre resistência a uma hegemonia ou a uma homogeneização, àquilo que Derrida chama de uma "homohegemonia". Ela entra sempre em resistência em relação à figura da dominação e, primeiramente, no campo da cultura. É contra a pretensão hegemônica da razão hegeliana - e portanto universal -, entendida como identidade absoluta a si mesmo, que a filosofia contemporânea entra em resistência. Ela se esforça, com efeito, renunciando ao fantasma de uma pureza original, para pensar uma identidade que não só acolhe uma alteridade mas ainda se deixa alterar por ela. Quer se trate de jazz ou de língua, de interpretação (Adorno e Patrick Willia$\mathrm{ms}$ ) ou de tradução (Walter Benjamin), quer se trate até mesmo de arte contemporânea e de sua relação com o Museu (Breton, Picasso, Malraux), é sempre na resistência que se constitui uma verdadeira identidade - que é recusa de fechamento em si mesmo. 
Abstract

Resistance is always resistance to hegemony or homogenization, to what Derrida called a "homohegemony." This concept always marks a resistance to figures of domination - particularly in the field of culture. It is against the hegemonic pretensions of Hegelian - and therefore universal - reason, understood as absolute identity to itself, that contemporary philosophy resists. Renouncing the phantasm of an original purity, contemporary philosophy endeavors, in effect, to think an identity that not merely welcomes an alterity but also allows itself to be altered by it. Be it with respect to jazz or language, interpretation (Adorno and Patrick Williams) or translation (Walter Benjamin), or even contemporary art and its relation to the Museum (Breton, Picasso, Malraux), it is always in resistance that is constructed true identity - which is a refusal of self-closure.

\section{Résumé}

La résistance est toujours résistance à une hégémonie ou à une homogénéisation, à ce que Derrida nomme une "homohégémonie". Elle entre toujours en résistance à la figure de la mâtrise - et d'abord dans le domaine de la culture. C'est contre la prétention hégémonique de la raison hégélienne - et donc de l'universel -, entendue comme l'identité absolue à soi, que la philosophie contemporaine entre en résistance. Elle s'efforce, en effet, renonçant au fantasme d'une pureté originaire, de penser une identité qui non seulement accueille une altérité mais se laisse altérer par elle. Qu'il s'agisse de jazz ou de langue, d'interprétation (Adorno et Patrick Williams) ou de traduction (Walter Benjamin), qu'il s'agisse même de l'art contemporain et de son rapport au $\mathrm{Mu}$ sée (Breton, Picasso, Malraux), c'est toujours dans la résistance que se constitue une véritable identité - qui est refus d'une clôture sur soi.
Key words: resistance; identity; alterity; language; culture; art.

Mots-clés : résistance; identité; altérité; langue; culture; art. 